\title{
The Definition of Density in General Relativity
}

\section{Ernst Fischer}

Stolberg, Germany

Email: e.fischer.stberg@t-online.de

How to cite this paper: Fischer, E. (2017) The Definition of Density in General Relativity. International Journal of Astronomy and Astrophysics, 7, 303-312. https://doi.org/10.4236/ijaa.2017.74025

Received: May 15, 2017

Accepted: December 24, 2017

Published: December 27, 2017

Copyright $\odot 2017$ by author and Scientific Research Publishing Inc. This work is licensed under the Creative Commons Attribution International License (CC BY 4.0).

http://creativecommons.org/licenses/by/4.0/

\begin{abstract}
According to general relativity the geometry of space depends on the distribution of matter or energy fields. The relation between the local geometrical parameters and the volume enclosed in given limits varies with the distribution of matter. Thus properties like particle number, mass or energy density, defined in the Euclidean tangent space, cannot be integrated to give conserved integral data like total number, mass or energy. To obtain integral conservation, a correction term must be added to account for the curvature of space. For energy this correction term is the equivalent of potential energy in Newtonian gravitation. With this correction the formation of black holes in the sense of singularities by gravitational collapse does no longer occur and the so called dark energy finds its natural explanation as potential energy of matter itself.
\end{abstract}

\section{Keywords}

Cosmology, Gravitational Collapse, Black Holes

\section{Introduction}

According to Einstein's theory of general relativity (GRT) motion of particles under the influence of gravitation is described by geodesic motion in a non-Euclidean space-time continuum. The deviations from Euclidean space are described by the field equations, which relate the local curvature to the distribution of matter or energy. In the last century observational tests have impressively confirmed the theoretical model: the aberration of light passing close to the sun, the perihelion shift of Mercury or other planets and the frequency shift of light by gravitational interaction. But all these observations were related to the motion of test particles or quanta in the gravitational field of a central mass, which means geodesic motion in a space-time of given metric.

In later years the model has been extended to situations, where more than one 
mass contribute to the metric as in orbiting and colliding double star systems. The correct description of the double pulsar systems and, more recently, of the metric deformation, caused by the final orbiting and collapse of binary "black holes" shows the validity of the GRT model.

But the situation is still more intricate, when the motion of continuously distributed matter is considered. In this case the vanishing of flow divergences of conserved properties must be expressed by a covariant formalism, adjusted to the curved metric. But there is the additional problem that the metric is no longer fixed. The local volume element depends on the curvature of space and thus on the matter distribution, so that the density, the amount of some quantity per volume, may change with the metric without there being any flow of matter or energy. There are two independent mechanisms, which may change the local density, flow through the boundaries and change of the volume element, when the curvature of space changes.

This fact has been left out of consideration in the textbooks of GRT with the argument that conservation laws are valid only locally (see e.g. [1]) or with the assumption that energy is somehow carried away by gravitational radiation (see e.g. [2]), when the geometry of space changes. But if we propose that there exist properties, like the number of particles within some predefined limits of space, which are generally conserved, we must take into account the fact that the relation between the local geometrical parameters and the volume elements is no longer fixed, but changes with the metric.

Thus to describe densities of conserved quantities like the number of particles (or their mass) we must take into consideration the change of volume by curvature, if we want to sum up or integrate their contribution to integral data like the total mass. Also the local definition of energy density, as we are accustomed from Euclidean geometry, does no longer represent an integrally conserved quantity. To guarantee integral particle number or energy conservation in GRT, the density, which we would measure in the flat space limit, can only be used as a reference value. In curved space it must be modified and corrected by a term, which depends on the curvature.

To demonstrate the problem of density definition, in the next section we will first discuss a very simplified example of matter distribution on a curved twodimensional surface. In the third section the inclusion of the curvature related effects into the energy tensor of GRT will be discussed. After that it will be shown that the occurrence of singular horizons by gravitational collapse is only a mathematical artefact, caused by the inaccurate definition of density. Finally possible consequences related to global cosmological models will be addressed.

\section{Density on a Curved Surface}

To demonstrate the problem that occurs in curved geometry with the definition of matter density and its integration to obtain the total mass, we consider a spherical surface with constant matter distribution. In Euclidean geometry we would 
describe such a surface by spherical coordinates centered at some point at distance $a$ from the surface.

Any point on the surface is defined then by its angular coordinates $\vartheta$ and $\varphi$. The line element on the surface is defined as

$$
\mathrm{d} s^{2}=a^{2}\left(\mathrm{~d} \vartheta^{2}+\sin ^{2} \vartheta \mathrm{d} \varphi^{2}\right)
$$

But in the framework of differential geometry, which is the mathematical basis of GRT, we cannot use coordinates defined at some point away from the surface. Instead we use local coordinates, defined only within the surface. We must define a radial parameter inside the surface to measure the distance from some reference point. Setting $r=a \sin \vartheta$ The line element reads

$$
\mathrm{d} s^{2}=h(r) \mathrm{d} r^{2}+r^{2} \mathrm{~d} \varphi^{2}
$$

with $h(r)=1 /\left(1-r^{2} / a^{2}\right)$. The radial parameter $r$ is not the distance from the reference point, measured along the curved surface, but the length of a circle at constant distance from this point, divided by $2 \pi$. Only in the limit of vanishing curvature it reduces to the radial coordinate. The area contained within a circle of length $2 \pi r_{0}$ is

$$
A=2 \pi \int_{0}^{r_{0}} \sqrt{h(r)} r \mathrm{~d} r
$$

and thus depends on the curvature parameter $a$. It increases with the curvature of the surface.

Assuming that the surface is covered with particles of given mass and we want to determine the total mass included in the circle of radius $r_{0}$, we cannot take the surface density as measured in flat geometry and integrate it over the curved surface, as this would not be a conserved quantity, when the curvature is changed. To define a conserved quantity, the density, as defined in the local Euclidean tangent system, should be corrected by a term, which accounts for the curvature of the surface. Instead of integrating the density $\rho_{0}$, defined in the limit of a flat surface, we must use the density $\rho=\rho_{0} / \sqrt{h(r)}$ to calculate the invariant total mass inside the circle of radius $r_{0}$. The Integral

$$
M=2 \pi \int_{0}^{r_{0}} \rho \sqrt{h(r)} r \mathrm{~d} r=2 \pi \int_{0}^{r_{0}} \rho_{0} r \mathrm{~d} r
$$

is invariant under the change of curvature. Generally speaking this means that we cannot maintain the accustomed definitions of matter or energy density from Euclidean space as local properties, when we want to derive conserved macroscopic data by integration.

\section{The Tensor of Energy Density}

In GRT the definition of energy density is still more intricate. Not only that space may be curved in three dimensions. Instead of a scalar matter density we have to handle with a tensorial property, which may contain contributions of matter, radiation and kinetic energy, and moreover the energy tensor itself is the source of 
deviations from Euclidean geometry. This dependence is described by the field equation

$$
R_{i j}-\frac{1}{2} R g_{i j}=\kappa T_{i j}
$$

which couples the geometry, expressed by the metric tensor $g_{i j}$ and its derivatives, contained in the Ricci tensor $R_{i j}$, to the energy tensor $T_{i j}$ (the constant $\kappa$ is defined as $8 \pi G / c^{2}, G$ is the gravitational constant). The question is: How must the local energy tensor be defined under the proposition that the energy fields represent properties, which are integrally conserved, when the metric is changed?

Regarding total energy as a conserved quantity, we cannot expect that the energy tensor, as we know it from Minkowski space-time, is valid in curved space, but we have to apply a correction to account for curvature. But to keep the tensorial character of GRT, the correction term must be independent of the choice of the coordinate basis. To preserve the covariance properties, it can only be formed by the metric tensor or quantities derived from it without differentiation and by the values of the local properties, which one would measure in the flat space limit. The flat space tensor $\hat{T}_{i j}$ has to be replaced by a quantity of the form

$$
T_{i j}=\hat{T}_{i j}+\lambda g_{i j}
$$

where $\lambda$ is a scalar function of locally defined parameters and tends to zero with vanishing curvature. The parameters which can be used to define $\lambda$ are the total energy density, that means the invariant trace of the energy tensor as defined in Minkowskian tangent space, $\hat{T}=\hat{T}_{i j} \hat{g}^{i j}$, and a quantity which expresses the relation between the volume elements of curved space and the corresponding Euclidean tangent space. The ratio of the three-space volume elements can be expressed by that of the spatial tensor densities $\sqrt{g^{(3)} / \hat{g}^{(3)}}$, where $g^{(3)}$ and $\hat{g}^{(3)}$ denote the spatial subdeterminants of the metric tensor in curved resp. Euclidean tangent space. Using these quantities, the function $\lambda$ must be defined as

$$
\lambda=\hat{T}\left(\sqrt{\frac{\hat{g}^{(3)}}{g^{(3)}}}-1\right)
$$

To understand the consequences of this correction, we consider a static system of spherical symmetry. In curved spherical systems the line element is generally described in the form

$$
\mathrm{d} s^{2}=-f(r, t) \mathrm{d} t^{2}+h(r, t) \mathrm{d} r^{2}+r^{2} \mathrm{~d} \Omega^{2}
$$

( $t$ is the time parameter, $r$ a radial parameter and $\mathrm{d} \Omega^{2}=\mathrm{d} \vartheta^{2}+\cos ^{2} \vartheta \mathrm{d} \varphi^{2}$ defines the element of solid angle $\mathrm{d} \Omega$ ). The radial parameter $r$, analog to the example of the last section, is defined as the length of a closed line at constant parameter $r$, divided by $2 \pi$. In the static case it is sufficient to consider the spatial part: 


$$
\mathrm{d} \sigma^{2}=h(r) \mathrm{d} r^{2}+r^{2}\left(\mathrm{~d} \vartheta^{2}+\cos ^{2} \vartheta \mathrm{d} \varphi^{2}\right)
$$

Using Equation (7) from Equation (6) we get

$$
T_{i j}=\hat{T}_{i j}+\hat{T}_{k l} \hat{g}^{k l}(1 / \sqrt{h(r)}-1) g_{i j}
$$

Physically the second term, which appears as a formal mathematical correction to account for the volume change by curvature, is nothing but the inclusion of potential energy in the energy balance. This can easily be shown considering the limiting case of the Schwarzschild solution [3], where the geometry is determined by a large central mass, so that the contribution of some matter density outside this central mass to the geometry is negligible.

Under these conditions the static field equations are reduced to

$$
\frac{1}{h r^{2}} \frac{\mathrm{d} h}{\mathrm{~d} r}+\frac{1}{r^{2}}\left(1-\frac{1}{h}\right)=0
$$

with the solution $h(r)=1 /\left(1-2 G M / c^{2} / r\right)$. ( $G$ is the gravitational constant and $M$ the mass of the central object) If there is some matter of density $\rho$ (as defined in flat Euclidean tangent space), the contribution to the energy tensor must be determined from Equation (10). The contribution to the component $T_{00}$ is given by

$$
T_{00}=\rho+\rho(1 / \sqrt{h(r)}-1) \approx \rho-\frac{\rho G M}{c^{2} r}
$$

The second term is just the potential energy, as we know it from Newtonian theory. The conserved quantity in GRT is the sum of matter energy and potential energy, as we are accustomed from Newtonian physics. When the action of gravity is expressed by the curvature of space, potential energy finds its place as a volume change by curvature.

There is, however, the difference that in GRT potential energy is described by a tensor. That means that there is not only a contribution to $T_{00}$, but also to the pressure term, which looks like a negative pressure. But this negative pressure exists in Newtonian theory, too. It is the negative pressure counteracting the positive kinetic atmospheric pressure of the gas, which is normally ascribed to the weight of the gas.

In any fluid with continuously distributed matter work has be done to expand the fluid against the mutual gravitational attraction of the constituent particles. That this tension or negative pressure scarcely can be observed under terrestrial conditions is due to the fact that the positive kinetic pressure normally is larger by many orders of magnitude.

The gravitational correction of energy density is negligible under terrestrial conditions. The factor $G M /\left(c^{2} r\right)$ on the surface of earth is of the order $10^{-9}$. The only effect, which can be expected, is that estimates of masses contain errors of this order. Only in the gravitational collapse of stars or galaxies deviations from Euclidean geometry of space may become important or in the global cosmological evolution, where curvature effects may be comparable to expansion 
effects. In the next section the influence of curvature corrections on the balance of collapsed systems will be discussed.

The results of the present section can be summed up as follows: Integral energy conservation in curved space can be obtained only if the densities of the constituting matter or energy fields are corrected by an additional term, representing the potential energy associated with curvature. If we express gravitational interaction by a change of geometry, we have to include potential energy in the definition of the energy density, which is the source of this change.

\section{Gravitational Collapse}

One of the most discussed questions in cosmology is the problem of collapse of stars or complete galaxy centers under the influence of gravitation, leading to white dwarfs, neutron stars and, according to the common view of GRT, even to situations, where no stable equilibrium state is possible, so that collapse ends in a singularity of space-time.

Static spherically symmetric solutions of the Einstein field equation are well known as the solutions of the TOV equation [4], which relates the internal matter and pressure distribution to the geometry of space. In this equation the diagonal elements of the energy tensor are expressed by an energy density $T_{00}=\rho$ and a homogeneous pressure $T_{i i}=P \quad(i=1,2,3)$. Integration of the field Equation (5) then leads to the formula (in units with $c=1$ and $G=1$ )

$$
\frac{\mathrm{d} P}{\mathrm{~d} r}=-(\rho+P) \frac{m(r)+4 \pi r^{3} P}{r^{2}(1-2 m(r) / r)}
$$

where the function $\mathrm{m}(\mathrm{r})$ is defined by the integral

$$
m(r)=4 \pi \int_{0}^{r} \rho r^{2} \mathrm{~d} r
$$

and is related to the radial curvature parameter $h(r)$ occurring in the solution of the field equation

$$
\frac{1}{r h^{2}} \frac{\mathrm{d} h}{\mathrm{~d} r}+\frac{1}{r^{2}}\left(1-\frac{1}{h}\right)=8 \pi \rho
$$

by $h(r)=1 /(1-2 m(r) / r)$.

The relation between the pressure and the matter density is assumed to be given by some equation of state, including various terms like kinetic pressure, radiation pressure and even quantum degeneracy pressure (at extreme densities). But in conventional treatment in the textbooks the density is always taken at its limiting flat space value. This leads to the result that the right hand side of Equation (13) becomes singular, when $2 m(r) / r$ is equal to 1 . This corresponds to the Schwarzschild radius $r_{s}$ of the geometry generated by some individual mass $m$.

In the derivation of the TOV equation it is assumed that the density of matter rest energy and of thermal kinetic energy (pressure) can be defined as conserved quantities independent of the curvature of space. But in a dynamical problem, where 
the geometry changes with time, at least the number of constituting particles should be conserved and also the total energy of the system.

To satisfy integral conservation of energy, instead of the flat space densities the corrected densities as defined by Equation (10) should be used. That means that the right hand side of Equation (15) depends implicitly on the parameter $h(r)$. The quantity $m(r)$ cannot be calculated by simple integration as in Equation (14), but must be determined from the differential equation

$$
\frac{\mathrm{d} m}{\mathrm{~d} r}=4 \pi r^{2} \rho \sqrt{1-2 m / r}
$$

with the boundary condition $m(0)=0$. With this redefinition the matter energy density $\rho$ at the Schwarzschild radius, defined in the local tangent space, just balances its own potential energy $\rho\left(\sqrt{1-r_{s} / r}-1\right)$, so that the effective contribution to the total energy remains finite.

Including potential energy into the energy balance in this way, a basic property of Newtonian gravity is recovered, the integral conservation of energy. Without inclusion of potential energy into the balance, gravitational collapse would be accompanied by a continuous gain of energy from the gravitational field. But in the geometrical concept of general relativity there exists no gravitational field which might possess energy. Gravitation is only a consequence of curvature. So this energy would be created from nothing. Only if we include potential energy of matter itself into the balance in form of the volume correction, conservation of energy is guaranteed also in systems of strong curvature. This leads to a basically different form of the solution to the balance equations.

This can be best demonstrated assuming as an example a system of constant density $\rho_{0}$ and radial extension $R$. It is reasonable to assume that in every real isolated matter distribution the condition $\mathrm{d} \rho / \mathrm{d} r<0$ holds, so that with constant $\rho=\rho_{0}$ the maximum deviation of $h(r)$ from unity will be obtained. In this case introducing a normalized coordinate $x=r / R$ with $8 \pi \rho_{0} R^{2}=1$ and the new variable $y=x / h$ Equation (15) can be written in the form

$$
\frac{\mathrm{d} y}{\mathrm{~d} x}=1-x^{3 / 2} y^{1 / 2}
$$

while without the potential energy term we have

$$
\frac{d y}{d x}=1-x^{2}
$$

Figure 1 shows the resulting function $h(x)$ for both equations. Without potential energy the solution exhibits a singularity at $x=\sqrt{3}$, while with potential energy $h(x)$ is finite for all $x$.

The solution of Equation (17) with $y(0)=0$ exhibits an extremum at $x=1.12$ with a maximum value $y_{\max }=0.71$. This value must be regarded as an upper boundary also for other density distributions as long as the condition $\mathrm{d} \rho / \mathrm{d} r \leq 0$ is valid. With other words: The Schwarzschild limit, which requires 


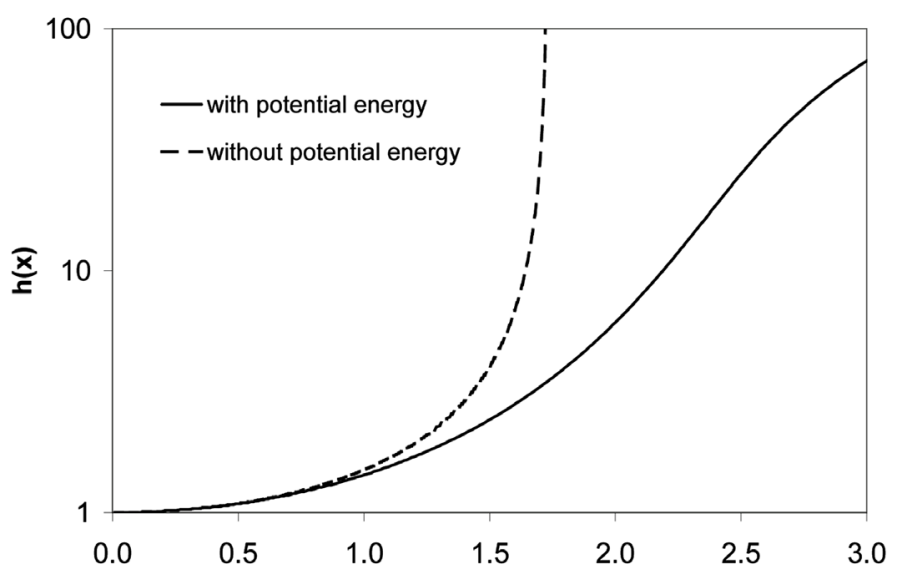

Figure 1. Radial metric parameter $h(x)$ as a function of the normalized coordinate $x=r / \sqrt{8 \pi \rho_{0}}$.

$y_{\max }=1$, can never be reached for any reasonable matter distribution. The Schwarzschild radius must be regarded as a purely mathematical quantity without relevance for real physical objects.

The fact that the quantity $(1-2 m(r) / r)$ can never reach zero is essential also for the solution of the pressure balance Equation (13). The denominator of the TOV equation is always positive. No infinite pressure is ever necessary to balance gravitational force. Due to the form of the potential energy term $\lambda(r) g_{i j}$ there is also a negative contribution of curvature to the pressure, but this does not change the general form of the solution.

The solution of the system of Equations (13) and (14) requires the knowledge of an equation of state, some functional relation between $\rho$ and $P$, just like in conventional modelling of stars, where potential energy is neglected. But there is no situation, where a static balance is impossible and collapse would proceed into a singular state. The only proposition for a stable equilibrium configuration is that $\rho$ and $P$ decrease monotonically from the center to the surface of the matter distribution.

In normal stars, where gravitational attraction is balanced by thermal pressure produced by thermonuclear reactions, pressure and density are always monotonic functions of the radial coordinate. Besides that, the influence of potential energy on the balance is negligibly small. This remains true also when the thermonuclear fuel is consumed and the star cools off by radiation. In this case the degeneracy pressure of electrons or neutrons takes over, to stabilize the star against further gravitational collapse. There may occur situations, as e.g. in white dwarfs, where the degeneracy pressure decreases with increasing density due to a phase transition. When electrons and protons recombine into neutrons, this may lead to instabilities and stellar explosions, as we know them as supernovas.

At extreme densities there may be further phase transitions by the supposed formation of hyperon or quark matter. There exists a broad spectrum of theoretical models regarding the equation of state under such extreme conditions, but fi- 
nally every collapsing object can end in an equilibrium state, where degeneracy pressure of the constituting particles balances gravitation. There is no upper mass or density limit, where infinite pressure would be required to reach equilibrium. No horizon exists, where accreted matter is inevitably lost from the surrounding space. Matter may collapse to extreme concentrations, but never to a "black hole" in the sense of a space-time singularity.

Collapsed systems are possible on all matter scales from individual stars to complete galaxies. They may interact with each other, combine into larger configurations or absorb incoming matter, but will always remain of finite size.

\section{Discussion}

In the last section we have demonstrated that to obtain integral energy conservation we have to take into account the fact that the relation between the path elements and the volume element is changed by curvature or, to express it in more physical way, to include potential energy in the energy tensor. Due to its negative contribution to the balance potential energy puts into question the energy condition that the quantity $\rho+3 P$ should be positive everywhere and for all physical fields. Just on this proposition the singularity theorems of Penrose [5] and Penrose and Hawking [2] are based. From the fact that gravity is always attractive they conclude that singularities are unavoidable in gravitational collapse. But when the effective mass energy per volume decreases due to the change of the volume elements by curvature and when this is accompanied by an increasing negative pressure, this conclusion is no longer valid.

Of course, mathematically singular solutions are possible, if we allow that arbitrarily large amounts of matter can be collected into arbitrarily small volumes. This is the same kind of singularity as we observe it in electromagnetism, if we regard an electron as a real point charge of zero spatial extension.

By now we have only discussed equilibrium configurations. In nature such idealized equilibria are scarcely reached. Most collapsing objects continuously accrete matter from surrounding space. Besides, there is no spherical symmetry. The objects may rotate and the inflow of matter may be concentrated to the rotational plane. Also magnetic fields can influence the motion of charged particles.

Thus during the formation of collapsing objects unstable situations may occur, leading to expulsion of the outer matter shell or to the nearly complete disruption of a star. But in a final state, when degeneracy pressure dominates the dynamics, kinetic pressure of incoming matter is negligible. It is only rotation of the complete system, which may influence the dynamical equilibrium. In this case pressure is no longer isotropic. Inflow of matter near the rotational plane will cause an outflow along the axis of rotation. The observed formation of cosmic matter jets from collapsed stars or active galaxy cores can be understood more easily, if we take as a fact that the "black hole" in the center is not a irrecoverable sink of matter on the other side of some horizon, but an accumulation of nuclear matter, which can be recycled into the universe under suitable conditions. This is of es- 
sential importance to understand the physics of the matter jets emerging from the supermassive "black holes" in the cores of active galaxies. Detailed modelling of these cores is still missing, but to understand them, the first requirement is that we start from the correct balance equations.

\section{Conclusions}

Neglecting potential energy in the balance equations appears as a general problem in the conventional form of general relativistic modelling. In the description of the global dynamics of the universe people are looking for the so called dark energy, which is necessary to bring the theoretical model into agreement with observations. This dark energy should be present throughout the universe, exhibit a negative pressure and a negative energy density comparable in order of magnitude to that of matter. Potential energy of matter itself just fulfills all these requirements. In a homogeneous solution of the Einstein equation it would look just like a cosmological constant, with the only difference that it is not a true constant, but varies with the matter density. Thus we do not need some additional mysterious dark energy to fulfil the balance.

By now we have no secure confirmation that general relativity delivers the correct description of the universe, but in the weak field limit it has successfully passed all observational tests. With the corrections discussed in this paper some of its shortcomings in the strong field limit, the existence of singularities, the existence of the mysterious dark energy and its agreement in magnitude with the matter density are automatically resolved. The only thing we have to do is, to accept the principle of energy conservation not only locally but also on macroscopic and global scale, independent of the geometry of space, simply by adjusting the definition of densities to the conditions of curved space.

\section{References}

[1] Wald, R.M. (1984) General Relativity. University of Chicago Press, Chicago. https://doi.org/10.7208/chicago/9780226870373.001.0001

[2] Hawking, S.W. and Ellis, G.F.R. (1973) The Large Scale Structure of Space-Time. Cambridge University Press, Cambridge. https://doi.org/10.1017/CBO9780511524646

[3] Schwarzschild, K. (1916) Sitzungsberichte der Koeniglich-Preussischen Akademie der Wissenschaften. Reimer, Berlin, 189-196.

[4] Oppenheimer, J.R. and Volkoff, G.M. (1939) On Massive Neutron Cores. Physical Revie, 55, 374. https://doi.org/10.1103/PhysRev.55.374

[5] Penrose, R. (1965) Gravitational Collapse and Space-Time Singularities. Physical Review Letters, 14, 57. https://doi.org/10.1103/PhysRevLett.14.57 